Canadian

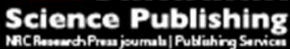

Applied Physiology, Nutrition, and Metabolism Physiologie appliquée, nutrition et métabolisme

\title{
Cardiorespiratory Demand of Acute Voluntary Cycling with Functional Electrical Stimulation in Individuals with Multiple Sclerosis with Severe Mobility Impairment
}

\begin{tabular}{|r|l|}
\hline Journal: & Applied Physiology, Nutrition, and Metabolism \\
\hline Manuscript ID & apnm-2017-0397.R1 \\
\hline Manuscript Type: & Article \\
\hline Date Submitted by the Author: & 28 -Jul-2017 \\
\hline $\begin{array}{r}\text { Complete List of Authors: } \\
\text { Is the invited manuscript for } \\
\text { consideration in a Special } \\
\text { Issue? : }\end{array}$ & $\begin{array}{l}\text { Edwards, Thomas; University of Illinois at Urbana-Champaign, Kinesiology } \\
\text { and Community Health; } \\
\text { Motl, Robert; University of Alabama at Birmingham }\end{array}$ \\
\hline Keyword: & $\begin{array}{l}\text { multiple sclerosis < nervous system, cardiorespiratory fitness, acute, } \\
\text { metabolic demand, functional electrical stimulation }\end{array}$ \\
\hline
\end{tabular}

\section{SCHOLARONE ${ }^{m}$ \\ Manuscripts}




\section{Cardiorespiratory Demand of Acute Voluntary Cycling with Functional Electrical}

Stimulation in Individuals with Multiple Sclerosis with Severe Mobility Impairment Thomas Edwards ${ }^{1}$, Robert W. Mott ${ }^{2}$, \& Lara A. Pilutti ${ }^{3}$

${ }^{1}$ Department of Kinesiology \& Community Health, University of Illinois at Urbana-Champaign, Urbana, Illinois, 61801, USA.

${ }^{2}$ Department of Physical Therapy, University of Alabama at Birmingham, 1705 University Blvd., Birmingham, AL, 35233-1212, USA.

${ }^{3}$ Interdisciplinary School of Health Sciences, Brain and Mind Research Institute, University of Ottawa, 200 Lees Avenue E250G, Ottawa, ON, K1N 6N5, Canada.

\section{Corresponding author:}

Lara A. Pilutti, Ph.D, Interdisciplinary School of Health Sciences, University of Ottawa 200 Lees Avenue, Ottawa, ON, K1N 6N5, Canada.

p: $613-562-5800$ ext. 6927

e : 1pilutti@uottawa.ca 


\section{ABSTRACT}

Background: Exercise training is one strategy for improving cardiorespiratory fitness (CRF) in multiple sclerosis (MS), however, few modalities are accessible for those with severe mobility impairment. Functional electrical stimulation (FES) cycling is one adapted exercise modality with the potential for improving CRF in people with severe MS.

Objectives: To characterize the cardiorespiratory response of acute voluntary cycling with FES in people with MS with severe mobility impairment, and to compare this response to passive leg cycling.

Methods: Eleven participants with MS that required assistance for ambulation completed a single bout of voluntary cycling with FES or passive leg cycling. Oxygen consumption $\left(\mathrm{VO}_{2}\right)$, heart rate (HR), work rate (WR), and ratings of perceived exertion (RPE) were recorded throughout the session.

Results: For the FES group, mean exercising $\mathrm{VO}_{2}$ was $8.7 \pm 1.8 \mathrm{ml} / \mathrm{kg} / \mathrm{min}$, or $63.5 \%$ of $\mathrm{VO}_{2 \text { peak }}$.

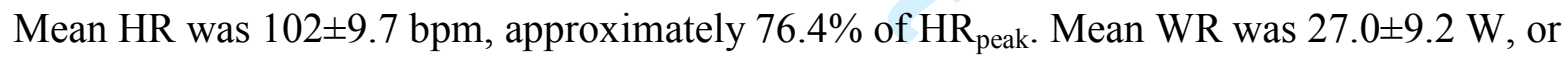
$57.3 \%$ of $\mathrm{WR}_{\text {peak }}$, and median RPE was 13.5 (IQR=5.5). Active cycling with FES was significantly $(p<.05)$ more intense than passive leg cycling based on $\mathrm{VO}_{2}, \mathrm{HR}, \mathrm{WR}$, and $\mathrm{RPE}$ during exercise.

Conclusions: Voluntary cycling with FES elicited an acute response that corresponded with moderate-to-vigorous intensity activity, suggesting that active cycling with FES can elicit a sufficient stimulus for improving CRF.

Key words: MULTIPLE SCLEROSIS, CARDIORESPIRATORY FITNESS, ACUTE, METABOLIC DEMAND, FUNCTIONAL ELECTRICAL STIMULATION 


\section{INTRODUCTION}

Cardiorespiratory fitness (CRF) is an important indicator of health for clinical and nonclinical populations, and low CRF is associated with increased risk for morbidity and mortality (Wei M et al. 1999, Motl and Pilutti 2013). Persons with multiple sclerosis (MS) have markedly lower CRF levels compared to controls without MS and CRF decreases as a function of increasing disability in individuals with MS (Motl and Goldman 2011, Motl and Pilutti 2013, Sandroff et al. 2013, Pilutti et al. 2015, Heine et al. 2016). Indeed, persons with severe MS disability have been reported to have a $52.6 \%$ lower peak oxygen consumption $\left(\mathrm{VO}_{2 \text { peak }}\right)$ compared with individuals with mild disability (Pilutti et al. 2015). Lower physical activity levels reported in individuals with mobility disability are likely an important contributor to physiological deconditioning (Klaren et al. 2013). Importantly, CRF has been associated with neurological disability, walking performance, brain structure, cognitive function, body composition, symptoms, and quality of life in persons with MS (Motl and Pilutti 2013, Sandroff et al. 2013, 2015a, 2015b, Pilutti et al. 2015, Motl et al. 2015, Heine et al. 2016). Consequently, intervention strategies that target and improve CRF in people with MS who have severe disability are of the utmost importance.

One approach that has been effective for improving CRF in persons with MS is exercise training. One meta-analysis reported that exercise training was associated with a moderate improvement ( $g=.47)$ in CRF in people with MS (Platta et al. 2016). However, most studies of exercise training have been limited to individuals with MS who have mild-to-moderate disability, often excluding those individuals with significant mobility impairment (Expanded Disability Status Scale $[$ EDSS] $\geq 5.5$ ). Alternative and accessible exercise approaches are needed to ensure that individuals with MS who have mobility disability have the opportunity to improve CRF and benefit from exercise training. 
One exercise modality that has been developed for individuals with mobility impairment is functional electrical stimulation (FES) cycling. FES cycling uses surface electrodes and mild electrical stimulation to evoke muscle contractions. These contractions are sequenced with a motorized cycle ergometer, simulating a cycling cadence (Reynolds et al. 2015, Pilutti et al. 2016) and could improve cycling performance. The superficial stimulation allows for greater recruitment of weakened muscles fibers during exercise, theoretically increasing overall oxygen consumption and the potential for adaptations with exercise training. If active cycling with FES results in an exercise intensity corresponding to moderate-to-vigorous physical activity it could represent a viable aerobic exercise training modality for improving CRF and managing physiological deconditioning for individuals with MS who have severe mobility impairment. Determining the exercise intensity of acute voluntary cycling with FES has important implications for the prescription of exercise training for individuals with MS with severe mobility impairment.

Herein, the purpose of this study was twofold: (i) to characterize the acute cardiorespiratory demand of a single bout of active cycling with FES in people with MS with severe mobility impairment; and (ii) to compare the cardiorespiratory demands of active cycling with FES to a single bout of passive leg cycling matched for exercise duration.

\section{METHODS}

\section{Participants}

Eleven participants from an ongoing trial of supervised voluntary cycling with FES were recruited to participate (Pilutti et al. 2016). Participants were part of a six-month exercise training intervention evaluating the efficacy of voluntary cycling with FES for improving 
walking ability, physiological fitness, and symptoms in individuals with severe MS disability (Pilutti et al. 2016). The participants were randomly allocated into one of two leg cycling conditions: (i) voluntary cycling with FES or (ii) passive leg cycling . Criteria for inclusion were: (i) between the ages of 18-64; (ii) a confirmed diagnosis of MS; (iii) use of unilateral or bilateral assistance for ambulation (EDSS score of 5.5-6.5); (iv) no history of a relapse within the past 30 days; (v) not currently participating in exercise on two or more days per week; (vi) asymptomatic (no known cardiovascular, pulmonary, or metabolic disease or symptoms suggestive of these conditions based on the Physical Activity Readiness Questionnaire (Bredin et al. 2013); (vii) not currently pregnant or plans to become pregnant during the trial; (viii) no contraindications for FES cycling (epilepsy, pacemaker, implanted defibrillator, fracture, or implanted screws/pins); (ix) physician approval for exercise testing and training.

\section{Outcome Measures}

\section{Clinical and Demographic Characteristics}

Height and weight were measured in the laboratory to the nearest $0.1 \mathrm{~cm}$ or $\mathrm{kg}$, respectively, using a scale with a stadiometer (Detecto, Webb City, MO). Disability status was determined through a clinically-administered EDSS (Kurtzke 1983) examination by a Neurostatus-certified assessor. Clinical and demographic characteristics were collected using a self-report questionnaire.

Peak Cardiorespiratory Fitness

Peak CRF was assessed using a symptom-limited incremental exercise protocol performed on a recumbent stepper (Nustep $\mathrm{T} 5^{\mathrm{XR}}$ recumbent stepper, Nustep Inc., Ann Arbor, MI) (Pilutti et al. 2015). The test began with a 1-minute warm-up at $15 \mathrm{~W}$ and the resistance was gradually increased by $5 \mathrm{~W}$ per minute until volitional fatigue (Pilutti et al. 2015). Expired gases 
were collected continuously using a two-way, non-rebreathable valve (Hans Rudolph Inc., Shawnee, KS) connected with an open circuit spirometry system (TrueOne 2400, Parvo Medics, Sandy, UT). Heart rate (HR; Polar Electro Oy, Kempele, Finland) and ratings of perceived exertion (RPE)(Borg 1982) were recorded every minute during the test. Peak power output was recorded from the recumbent stepper and expressed in Watts (W). Peak cardiorespiratory capacity $\left(\mathrm{VO}_{2 \text { peak }}\right)$ was determined when at least one of the following criteria were recorded: (i) respiratory exchange ratio $(\mathrm{RER}) \geq 1.10$; (ii) $\mathrm{HR}_{\text {peak }}$ within $10 \mathrm{bpm}$ of age-predicted maximum (i.e., 220-age); or (iii) RPE $\geq 17$ (Pilutti et al. 2015).

Acute Cycling Session

Participants completed a submaximal bout of leg cycling exercise on an RT300 cycle ergometer (Restorative Therapies Inc, Baltimore, MD). The participants in the FES cycling group received electrical stimulation during voluntary leg cycling via self-adhering surface electrodes placed over muscle groups of the lower extremities (quadriceps, hamstrings, and gluteals). The stimulation parameters were as follows: waveform symmetric biphasic, phase duration of $250 \mathrm{~ms}$, and pulse rate of 50 pulses per second. The intensity of leg muscle stimulation was adjusted per muscle group according to each participant's sensory tolerance. Participants were instructed to maintain a cycling cadence of $\sim 50 \mathrm{rpm}$ during the acute cycling session and pedaling resistance was automatically adjusted by the RT300 to maintain this cycling cadence. Exercise intensity was based on participants' individually determined heart rate reserve.

The participants in the passive cycling condition did not wear the electrodes and did not receive electrical stimulation. A pedaling cadence of $\sim 50 \mathrm{rpm}$ was generated entirely by the electric motor of the cycle ergometer. Continuous gas exchange and HR were measured during the entire session using the same system as for the peak CRF test. RPE was recorded every 
minute by a member of the research team (Borg 1982). Lastly, work rate (WR; expressed in Watts [W]), cycling resistance (expressed in Newton meters [Nm]), and the percentage of stimulation received, relative to maximum stimulation tolerated by each participant (\%MaxStim), were recorded continuously by the RT300 cycle ergometer and reported for describing the cycling session parameters.

\section{Protocol}

All procedures were approved by a University Institutional Review Board and participants provided written informed consent. The participants were informed of the possible risks and benefits during the informed consent process. Participants completed two testing sessions separate by approximately one week. At the first testing session, participants reported to the laboratory and completed the symptom-limited cardiopulmonary exercise test. This enabled the intensity of the acute session to be expressed as a percentage of peak values. At the second session, participants completed the acute cycling protocol (i.e., FES or passive leg cycling). Both the symptom-limited cardiopulmonary exercise test and acute cycling occurred within one week of midpoint (i.e., 3-months) testing of the 6-month trial. The submaximal acute exercise protocol consisted of four phases: (i) a five-minute period of monitored rest with no physical exertion (RE); (ii) a one minute transition/warmup period (WU); (iii) a 15-minute period of active cycling (AC); and (iv) a five-minute cool down period (CD). The acute exercise protocol is graphically presented in Figure 1.

\section{Data Analysis}

Data analysis was performed using IBM SPSS Statistics (Version 22.0, IBM Corp., Armonk, NY). Descriptive statistics were used to summarize the demographic, clinical, peak and acute cardiorespiratory response variables. The acute response variables were averaged per 
minute across the entire session and expressed as mean values for the RE, AC, and CD phases per cycling condition. The acute response was further expressed as a percentage of peak values obtained from the cardiopulmonary exercise test for $\mathrm{VO}_{2}, \mathrm{HR}$ and $\mathrm{WR}$, as these variables are commonly used indicators of exercise intensity. Comparisons between the FES cycling and passive cycling groups were made using independent samples $t$-tests, $c h i$-square tests, and oneway analysis of variance (ANOVA) with post-hoc Bonferroni corrections. Statistical significance was set at $p<.05$.

\section{RESULTS}

\section{Characteristics of the Participants}

The characteristics of the participants in the FES and passive cycling conditions are reported in Table 1. There were no significant differences in height, weight, disability, or disease duration between the two conditions. There was a significant difference in age between the FES cycling and passive cycling condition, such that the FES cycling group was older than the passive cycling group ( $p=.03$ ). Consequently, analysis of covariance (ANCOVA) were performed using age as a covariate in all subsequent comparisons by condition. There were no significant differences in the peak cardiorespiratory response between the FES cycling and passive leg cycling condition (all $p>.05$ ).

\section{Acute Cardiorespiratory Response}

Figure 2 provides a graphical presentation of the mean physiological response variables for the FES cycling group per minute during the submaximal exercise session. Mean oxygen consumption $\left(\mathrm{VO}_{2}\right)$ increased by $190 \%$ from $3.0 \pm 0.6 \mathrm{ml} / \mathrm{kg} / \mathrm{min}$ during $\mathrm{RE}$ to $8.7 \pm 1.8 \mathrm{ml} / \mathrm{kg} / \mathrm{min}$ 
(63.5\% of $\mathrm{VO}_{2 \text { peak }}$ ) during $\mathrm{AC} . \mathrm{VO}_{2}$ then returned to $5.9 \pm 1.3 \mathrm{ml} / \mathrm{kg} / \mathrm{min}$ during the $\mathrm{CD}$. Mean HR increased by $50 \%$ from $68 \pm 12.1 \mathrm{bpm}$ during RE to $102 \pm 9.7 \mathrm{bpm}\left(76.4 \%\right.$ of $\left.\mathrm{HR}_{\text {peak }}\right)$ during $\mathrm{AC}$, then decreased to $91 \pm 14.3 \mathrm{bpm}$ in CD. During the AC phase, mean WR was $27.0 \mathrm{~W}$ (57.3\% $\left.\mathrm{WR}_{\text {peak }}\right)$, and median RPE reported was $13.5(\mathrm{IQR}=5.5)$. Mean resistance on the cycle was 4. $7 \pm 0.2 \mathrm{Nm}$, and the mean stimulation received was $35.3 \pm 26.6 \%$ of maximally tolerated stimulation.

FES Cycling vs Passive Leg Cycling Response

The mean physiological responses recorded during the AC phase of FES cycling and passive leg cycling are presented in Table 2 . There was a significant difference in mean $\mathrm{VO}_{2}$ $(p=.001), \operatorname{HR}(p=.002)$, WR $(p<.001)$, and RPE $(p<.003)$, such that mean values were higher during FES cycling compared to passive cycling based on ANCOVA. The mean RER recorded during FES cycling was greater than during passive cycling; however, this difference was not statistically significant $(p=.08)$.

When examining group differences based on the percentage of peak cardiorespiratory test values, the FES cycling condition achieved a significantly higher relative mean $\mathrm{VO}_{2}$, compared to the passive leg cycling group $(p=.007)$. Mean HR was higher in response to FES cycling compared to the passive leg cycling; however, this difference was not statistically significant $(p=.07)$. Lastly, The FES cycling group achieved a significantly higher mean WR expressed as a percentage of peak values, compared to the passive leg cycling group (both $p<.001$ ).

\section{DISCUSSION}


We conducted the first study to characterize the acute cardiorespiratory demands of active cycling with FES in people with MS with severe mobility impairment, and furthered compared this response to passive leg cycling. This is important for identifying if voluntary cycling with FES represents a potent enough stimulus (i.e., stressor) for yielding changes in CRF (i.e., adaptations) over time with training. Considering traditional indicators of exercise intensity, FES cycling exercise corresponded to an intensity of $63.5 \%, 76.4 \%$, and $57.3 \%$ of $\mathrm{VO}_{2 \text { peak, }}, \mathrm{HR}_{\text {peak }}$, and $\mathrm{WR}_{\text {peak }}$, respectively, and as expected active cycling with FES was more intense than passive leg cycling.

The American College of Sport Medicine (ACSM) recommends an exercise intensity of $40-85 \%$ of $\mathrm{VO}_{2 \text { peak }}$ or $70-90 \%$ of $\mathrm{HR}_{\text {peak }}$ (MVPA) for improving CRF in adults (Pollock et al. 1998, American College of Sports Medicine 2013). Furthermore, individuals with low CRF $\left(\mathrm{VO}_{2 \text { peak }}<40 \mathrm{ml} / \mathrm{kg} / \mathrm{min}\right)$, such as those with severe MS, can experience improvements in CRF at even lower exercise intensities $\left(\sim 30 \% \mathrm{VO}_{2 \text { peak }}\right)$ (Pollock et al. 1998, American College of Sports Medicine 2013). In the current study, participants were exercising at an intensity that satisfied the common criteria for attaining MVPA. This demonstrates that active cycling with FES is an adapted exercise modality that is capable of providing a sufficient exercise stimulus for improving $\mathrm{CRF}$, and should be considered an aerobic exercise training modality for managing physiological deconditioning in people with MS who have severe mobility impairment.

This is particularly important for individuals with MS at the upper end of the disability spectrum who experience significant physiological deconditioning. Of note, the exercise intensity achieved in response to active cycling with FES in the current study is consistent with intensities reported in previously aerobic exercise training studies in people with MS (LatimerCheung et al. 2013). This suggests that people with MS with mobility disability are capable of 
achieving similar intensities of aerobic exercise training as individuals with mild-to-moderate MS, and importantly, this intensity of exercise training is sufficient for improving CRF.

As expected, the cardiorespiratory response to voluntary cycling with FES was significantly more intense than that observed in response to passive cycling alone, suggesting an important contribution of voluntary leg cycling, added electrical stimulation, or the combination of both components. Interestingly, the mean exercising WR achieved in response to voluntary cycling with FES was $27.0 \mathrm{~W}$, or $57.3 \%$ of $\mathrm{WR}_{\text {peak. }}$ This is noticeably higher than previously reported WR values attained during FES cycling protocols in people with MS who have severe disability. One study reported a mean WR of 5.2W during a single FES cycling session (Fornusek and Hoang 2014). Another study reported a mean WR of 4.45W during a 30-minute FES cycling session (Backus et al. 2016). The goal of each session was to maintain a pedal cadence of 35-50 rpm. If a participant was unable to do so the stimulation automatically turned off and the FES cycle went into passive mode. It is important to note that both of these studies included participants that were nonambulatory and experienced greater symptomology, likely explaining the discrepancy in WR between the current study and the previous research.

The combination of voluntary leg cycling exercise with supplementary stimulation might enable greater exercise intensities and longer durations of exercise to be achieved (Fornusek and Hoang 2014). This approach is ideal for individuals with MS with severe disability as it would maximize the volume of exercise that can be accomplished, thus promoting greater physiological improvements. The supplementary neuromuscular stimulation provided from FES further has important benefits for people with MS with severe disability. It has been reported that individuals with MS have compromised muscular strength and motor-unit recruitment compared to healthy controls, despite similar muscle cross sectional area ( $\mathrm{Ng}$ et al. 2004). This impairment 
becomes more severe as disability increases (Ng et al. 2004, Pilutti et al. 2015). Such impairments in muscular function may be overcome by using neuromuscular stimulation in combination with leg cycling exercise to increasing overall muscle activation, allowing for greater motor-unit recruitment, muscle mass involvement, and force production (Reynolds et al. 2015). This supports the use of voluntary cycling with FES a potentially valuable exercise training modality for individuals with severe MS disability.

Another aspect of FES cycling that should be considered is the metabolic efficiency. To date, few studies have examined the acute metabolic demands of FES cycling. One study compared the metabolic efficiency (caloric equivalent of work performed/aerobic expenditure $\mathrm{x}$ 100) of FES cycling performed by participants with SCI to voluntary leg cycling in able bodied (AB) controls (Glaser et al. 1989). That study demonstrated that participants with SCI had cycling efficiencies ranging from 2-14\%, while AB participants had efficiencies ranging from 4$34 \%$. That study demonstrated that $\mathrm{VO}_{2}$ was higher during FES cycling compared to voluntary cycling, despite an equivalent WR. Another study compared the metabolic efficiency of FES cycling to voluntary cycling within a group of AB individuals (Hunt et al. 2013). That study demonstrated that FES cycling caused an increase in $\mathrm{VO}_{2}$ during exercise and determined that FES cycling was roughly half as metabolically efficient as voluntary cycling (Glaser et al. 1989, Hunt et al. 2013). These reported inefficiencies are likely a result of suboptimal biomechanics that occurs with FES cycling. External neuromuscular stimulation causes crude recruitment of the muscle groups, lack of synergistic and antagonistic joint control, mixed muscle fiber recruitment, and adverse muscle activation timing, all of which contribute to reduced metabolic/biomechanical efficiency during cycling (Sinclair et al. 1996, Gregory and Bickel 2005, Bickel et al. 2011). This apparent inefficiency may be beneficial in that it increases the 
energetic demand associated with this task, ultimately contributing to a more intense exercise stimulus and promoting physiological adaptations (Glaser et al. 1989).

\section{LIMITATIONS}

There are several important limitations of this study that must be considered. The sample size of the study was relatively small which may limit the applicability of the findings. Another limitation is the evaluation of a single exercise modality (RT 300 leg cycle). It would be valuable to compare the acute cardiorespiratory response using a variety of different adapted exercise modalities with and without FES, as well as through the modulation of voluntary and passive cycling conditions. This would allow for a greater understanding of the contribution of the neuromuscular stimulation and the voluntary exercise components. Finally, the peak cardiorespiratory exercise test and the acute cycling session were performed on two different exercise modalities. This could limit the accuracy of the physiological response expressed as a percentage of peak values.

\section{CONCLUSIONS}

We determined that voluntary cycling with FES elicits a cardiorespiratory stimulus that corresponds to MVPA and that FES cycling is more intense than passive leg cycling. The combination of voluntary leg cycling and supplementary neuromuscular stimulation can assist individuals with severe MS to achieve higher exercise training intensities, and consequently, could improve physiological fitness (i.e., CRF). Voluntary cycling with FES is an accessible, adapted exercise modality that can be used by people with MS with severe disability in the home 
or community setting, and should be considered as an option for aerobic exercise training in this population.

\section{CONFLICT OF INTREST}

None declared.

\section{ACKNOWLEDGMENTS}

This study was supported, in part, by the Multiple Sclerosis Society of Canada [EGID 2665], National Multiple Sclerosis Society [PR-1411-0209] and the Consortium of Multiple Sclerosis Centers. 


\section{REFERENCES}

American College of Sports Medicine. 2013. ACSM's Guidelines for Exercise Testing and Prescription. In Ninth edition. LWW, Philadelphia.

Backus, D., Burdett, B., Hawkins, L., Manella, C., McCully, K., and Sweatman, M. 2016. Pilot study of outcomes after functional electrical stimulation cycle training in individuals with multiple sclerosis who are nonambulatory. Int. J. MS Care. doi:10.7224/1537-2073.2015036.

Bickel, C.S., Gregory, C.M., and Dean, J.C. 2011. Motor unit recruitment during neuromuscular electrical stimulation: a critical appraisal. Eur. J. Appl. Physiol. 111(10): 2399-2407. doi:10.1007/s00421-011-2128-4.

Borg, G. 1982. Psychophysical bases of perceived exertion. - PubMed - NCBI. Med. Sci. Sports Exerc. 14(5): 377-381.

Bredin, S.S.D., Gledhill, N., Jamnik, V.K., and Warburton, D.E.R. 2013. PAR-Q+ and ePARmed-X+ New risk stratification and physical activity clearance strategy for physicians and patients alike. Can. Fam. Physician, 59(3): 273-277. 
Fornusek, C., and Hoang, P. 2014. Neuromuscular electrical stimulation cycling exercise for persons with advanced multiple sclerosis. J. Rehabil. Med. 46(7): 698-702. doi:10.2340/16501977-1792.

Glaser, R.M., Figoni, S.F., Hooker, S.P., Rodgers, M.M., Ezenwa, B.N., Suryaprasad, A.G., Gupta, S.C., and Mathews, T. 1989. Efficiency of FNS leg cycle ergometry. In Engineering in Medicine and Biology Society, 1989. Images of the Twenty-First Century., Proceedings of the Annual International Conference of the IEEE Engineering in. pp. 961-963 vol.3. doi:10.1109/IEMBS.1989.95639.

Gregory, C.M., and Bickel, C.S. 2005. Recruitment patterns in human skeletal muscle during electrical stimulation. Phys. Ther. 85(4): 358-364.

Heine, M., Wens, I., Langeskov-Christensen, M., Verschuren, O., Eijnde, B.O., Kwakkel, G., and Dalgas, U. 2016. Cardiopulmonary fitness is related to disease severity in multiple sclerosis. Mult. Scler. J. 22(2): 231-238. doi:10.1177/1352458515581437.

Hunt, K.J., Hosmann, D., Grob, M., and Saengsuwan, J. 2013. Metabolic efficiency of volitional and electrically stimulated cycling in able-bodied subjects. Med. Eng. Phys. 35(7): 919925. doi:10.1016/j.medengphy.2012.08.023. 
Klaren, R.E., Motl, R.W., Dlugonski, D., Sandroff, B.M., and Pilutti, L.A. 2013. Objectively quantified physical activity in persons with multiple sclerosis. Arch. Phys. Med. Rehabil. 94(12): 2342-2348. doi:10.1016/j.apmr.2013.07.011.

Kurtzke, J.F. 1983. Rating neurologic impairment in multiple sclerosis: an expanded disability status scale (EDSS). Neurology, 33(11): 1444-1452.

Latimer-Cheung, A.E., Pilutti, L.A., Hicks, A.L., Martin Ginis, K.A., Fenuta, A.M., MacKibbon, K.A., and Motl, R.W. 2013. Effects of exercise training on fitness, mobility, fatigue, and health-related quality of life among adults with multiple sclerosis: A systematic review to inform guideline development. Arch. Phys. Med. Rehabil. 94(9): 1800-1828.e3. doi:10.1016/j.apmr.2013.04.020.

Motl, R.W., and Goldman, M. 2011. Physical inactivity, neurological disability, and cardiorespiratory fitness in multiple sclerosis. Acta Neurol. Scand. 123(2): 98-104. doi:10.1111/j.1600-0404.2010.01361.x.

Motl, R.W., and Pilutti, L.A. 2013. The importance of physical fitness in multiple sclerosis. J. Nov. Physiother. 3(2): 141-147. doi:10.4172/2165-7025.1000141.

Motl, R.W., Pilutti, L.A., Hubbard, E.A., Wetter, N.C., Sosnoff, J.J., and Sutton, B.P. 2015. Cardiorespiratory fitness and its association with thalamic, hippocampal, and basal 
ganglia volumes in multiple sclerosis. NeuroImage Clin. 7: 661-666.

doi:10.1016/j.nicl.2015.02.017.

Ng, A.V., Miller, R.G., Gelinas, D., and Kent-Braun, J.A. 2004. Functional relationships of central and peripheral muscle alterations in multiple sclerosis. Muscle Nerve, 29(6): 843852. doi:10.1002/mus.20038.

Pilutti, L.A., Motl, R.W., Edwards, T.A., and Wilund, K.R. 2016. Rationale and design of a randomized controlled clinical trial of functional electrical stimulation cycling in persons with severe multiple sclerosis. Contemp. Clin. Trials Commun. 3: 147-152. doi:10.1016/j.conctc.2016.05.005.

Pilutti, L.A., Sandroff, B.M., Klaren, R.E., Learmonth, Y.C., Platta, M.E., Hubbard, E.A., Stratton, M.B., and Motl, R.W. 2015. Physical fitness assessment across the disability spectrum in persons with multiple sclerosis: A comparison of testing modalities. J. Neurol. Phys. Ther. 39: 1-9. doi:10.1097/NPT.0000000000000099.

Platta, M.E., Ensari, I., Motl, R.W., and Pilutti, L.A. 2016. Effect of Exercise Training on Fitness in Multiple Sclerosis: A Meta-Analysis. Arch. Phys. Med. Rehabil. 97(9): 1564-1572. doi:10.1016/j.apmr.2016.01.023. 
Pollock, M., Gaesser, G., and Butcher, J. 1998. The recommended quantity and quality of exercise for developing and maintaining cardiorespiratory and muscular fitness, and flexibility in healthy adults. Med. Sci. Sports Exerc. 30(6): 975-991.

Reynolds, M.A., McCully, K., Burdett, B., Manella, C., Hawkins, L., and Backus, D. 2015. Pilot study: Evaluation of the effect of functional electrical stimulation cycling on muscle metabolism in nonambulatory people with multiple sclerosis. Arch. Phys. Med. Rehabil. 96(4): 627-632. doi:10.1016/j.apmr.2014.10.010.

Sandroff, B.M., Klaren, R.E., and Motl, R.W. 2015a. Relationships among physical inactivity, deconditioning, and walking impairment in persons with multiple sclerosis. J. Neurol. Phys. Ther. 39(2): 103-110. doi:10.1097/NPT.0000000000000087.

Sandroff, B.M., Pilutti, L.A., Benedict, R.B., and Motl, R.W. 2015b. Association between physical fitness and cognitive function in multiple sclerosis: does disability status matter? Neurorehabil. Neural Repair, 29(3): 214-223. doi:10.1177/1545968314541331.

Sandroff, B.M., Sosnoff, J.J., and Motl, R.W. 2013. Physical fitness, walking performance, and gait in multiple sclerosis. J. Neurol. Sci. 328(1-2): 70-76. doi:10.1016/j.jns.2013.02.021. 
Sinclair, P.J., Davis, G.M., Smith, R.M., Cheam, B.S., and Sutton, J.R. 1996. Pedal forces produced during neuromuscular electrical stimulation cycling in paraplegics. Clin. Biomech. 11(1): 51-57.

Wei, M., Kampert, J.B., Barlow, C.E., Nichaman, M.Z., Gibbons, L.W., Paffenbarger, R.S., et al. 1999. Relationship between low cardiorespiratory fitness and mortality in normal-weight, overweight, and obese men. JAMA, 282(16): 1547-1553. doi:10.1001/jama.282.16.1547. 
Table 1: The demographic, clinical and peak cardiorespiratory fitness variables for the FES cycling and passive leg cycling groups.

Values are reported as means (SD), unless specified otherwise.

\begin{tabular}{|c|c|c|c|}
\hline Variable & FES Cycling $(n=6)$ & Passive Cycling $(n=5)$ & $p$-value \\
\hline \multicolumn{4}{|c|}{ Demographic and Clinical } \\
\hline Age (years) & $58(6.0)$ & $47.8(7.4)$ & $.03^{*}$ \\
\hline Height (cm) & $160.8(9.8)$ & $165.4(14.1)$ & .54 \\
\hline Weight (kg) & $68.8(17.1)$ & $95.5(42.7)$ & .19 \\
\hline EDSS, mdn (IQR) & $6.25(.63)$ & $6.0(.75)$ & .80 \\
\hline MS duration (years) & $20.8(7.8)$ & $21.0(7.3)$ & .97 \\
\hline \multicolumn{4}{|c|}{ Peak Cardiorespiratory Exercise Response } \\
\hline $\mathrm{VO}_{2 \text { peak }}(\mathrm{ml} / \mathrm{kg} / \mathrm{min})$ & $13.9(3.6)$ & $14.1(6.7)$ & .89 \\
\hline $\mathrm{HR}_{\text {peak }}(\mathrm{bpm})$ & $135.7(24.9)$ & $144.2(26.3)$ & .48 \\
\hline $\mathrm{WR}_{\text {peak }}(\mathrm{W})$ & $46.7(11.3)$ & $80.0(49.6)$ & .38 \\
\hline $\mathrm{RER}_{\text {peak }}$ & $1.2(0.4)$ & $1.1(0.1)$ & .54 \\
\hline $\mathrm{RPE}_{\text {peak }}, \mathrm{mdn}(\mathrm{IQR})$ & $17.0(4.25)$ & $15.0(5.5)$ & .78 \\
\hline
\end{tabular}


Abbreviations: $\mathrm{VO}_{2}$, volume of oxygen consumption; $\mathrm{HR}$, heart rate; WR, work rate; RER, respiratory exchange ratio; RPE, rating of perceived exertion.

*Indicates significant difference between groups at the $p<.05$ level. 
Table 2: The average physiological response at rest and during the active cycling phase of the acute exercise session for the FES cycling and passive leg cycling groups. Values are reported as mean (SD) unless specified otherwise.

\begin{tabular}{|c|c|c|c|}
\hline Variable & FES Cycling $(n=6)$ & Passive Cycling $(n=5)$ & $p$-value \\
\hline \multicolumn{4}{|l|}{ Resting Variables } \\
\hline $\mathrm{VO}_{2}(\mathrm{ml} / \mathrm{kg} / \mathrm{min})$ & $3.0(0.6)$ & $2.5(0.8)$ & 0.25 \\
\hline HR (bpm) & $68.4(12.1)$ & $75.9(4.4)$ & 0.22 \\
\hline \multicolumn{4}{|l|}{ Exercise Variables } \\
\hline $\mathrm{VO}_{2}(\mathrm{ml} / \mathrm{kg} / \mathrm{min})$ & $8.7(1.8)$ & $3.1(.58)$ & $.001 *$ \\
\hline HR (bpm) & $102.0(9.7)$ & $77.6(5.6)$ & $.002 *$ \\
\hline WR (W) & $27.0(9.2)$ & $.34(.63)$ & $<.001 *$ \\
\hline RER & $.93(0.1)$ & $.85(0.1)$ & .08 \\
\hline RPE, mdn (IQR) & $13.5(5.5)$ & $6.0(1.5)$ & $0.03 *$ \\
\hline \multicolumn{4}{|c|}{ Percentage of Peak Variable } \\
\hline$\% \mathrm{VO}_{2 \text { peak }}$ & $63.5(9.7)$ & $26.9(13.1)$ & $.007 *$ \\
\hline$\% \mathrm{HR}_{\text {peak }}$ & $76.4(9.7)$ & $55.5(12.9)$ & .07 \\
\hline$\% \mathrm{WR}_{\text {peak }}$ & $57.3(12.9)$ & $.43(.74)$ & $<.001^{*}$ \\
\hline
\end{tabular}


Abbreviations: $\mathrm{VO}_{2}$, volume of oxygen consumption; $\mathrm{HR}$, heart rate; WR, work rate; RER, respiratory exchange ratio; RPE, rating of perceived exertion.

*Indicates significant difference between groups at the $p<.05$ level. 


\section{FIGURE CAPTION}

Figure 1.

Graphical representation of the exercise protocol.

Figure 2.

Graphical representation of the continuous physiological response of the FES group during the acute exercise session.

Abbreviations: RE, resting phase; WU, Warmup phase; AC, Active cycling phase; CD cooldown phase. 


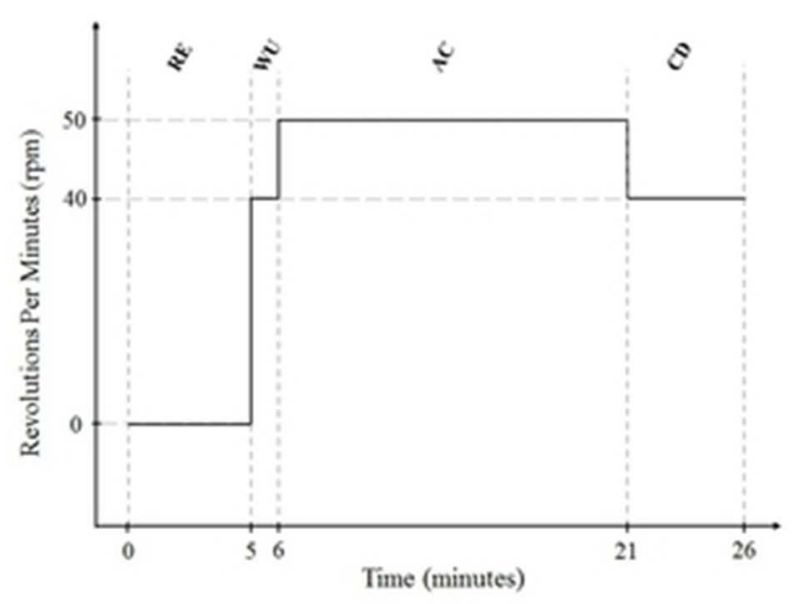

Figure 1

$25 \times 19 \mathrm{~mm}(300 \times 300 \mathrm{DPI})$ 

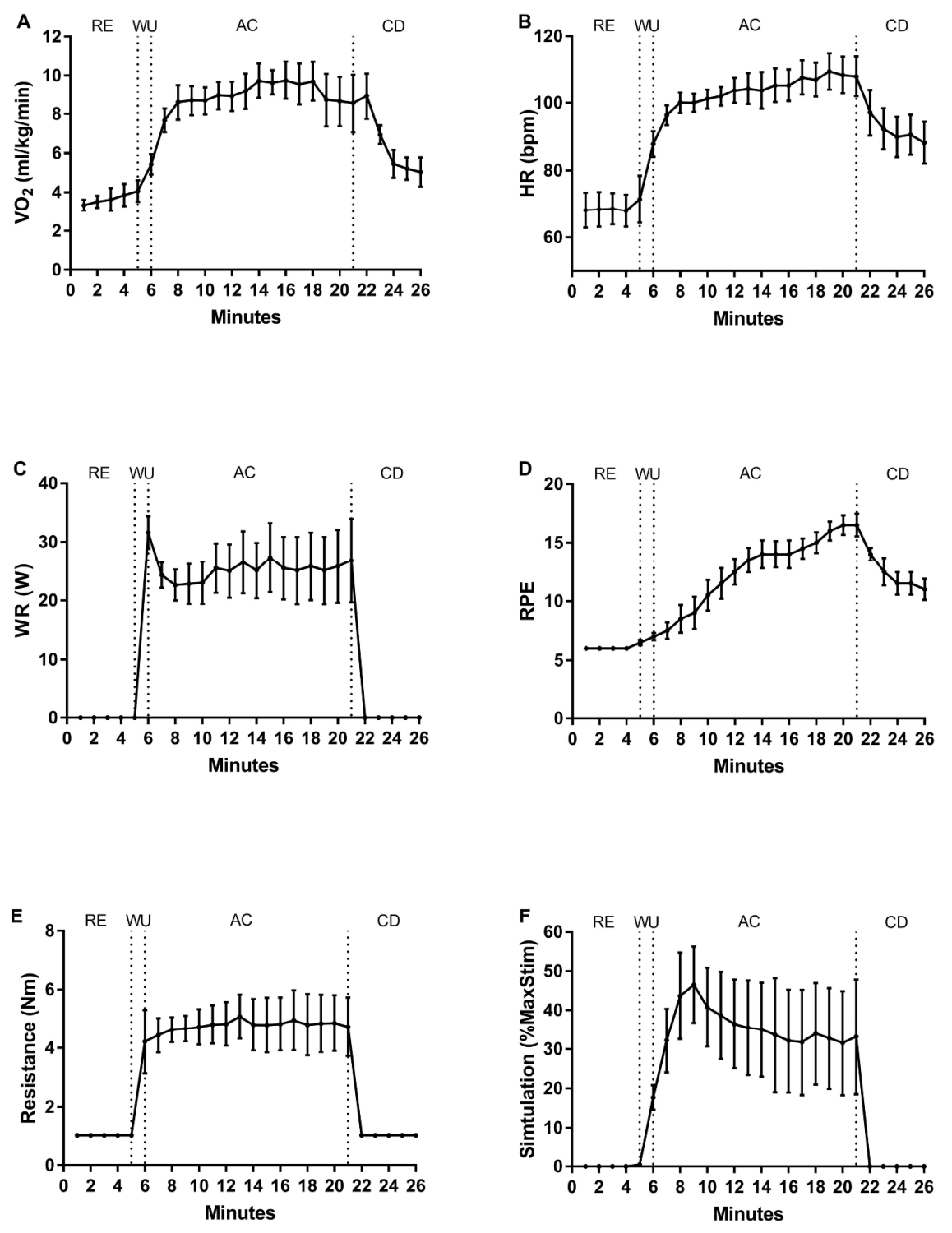

Figure 2

$245 \times 308 \mathrm{~mm}(300 \times 300$ DPI $)$ 